\title{
Unusual frequency dependence of the integrated profile of the millisecond pulsar PSR J2145-0705
}

\author{
A.D.Kuzmin and B.Ya.Losovski \\ Astro Space Center of Lebedev Physical Institute, Russia
}

We report on observations and frequency dependence analysis of the integrated profile of the millisecond pulsar PSR J2145-0705. Observations were performed at $102 \mathrm{MHz}$ with the BSA radio telescope in Pushchino.

To analyze the frequency dependence of the integrated profile we supplemented our data with high frequency measurements by Bailes et al. (unpublished). For frequency dependence analysis we used the Gaussian fit separation method of presentation the integrated pulse profile as a superposition of Gaussian-shaped individual components (Wu, Xu and Rankin 1992; Foster, Fairhead and Backer 1991). A good fit is provided by four component structure, suggested by Bailes et al. 1994 .

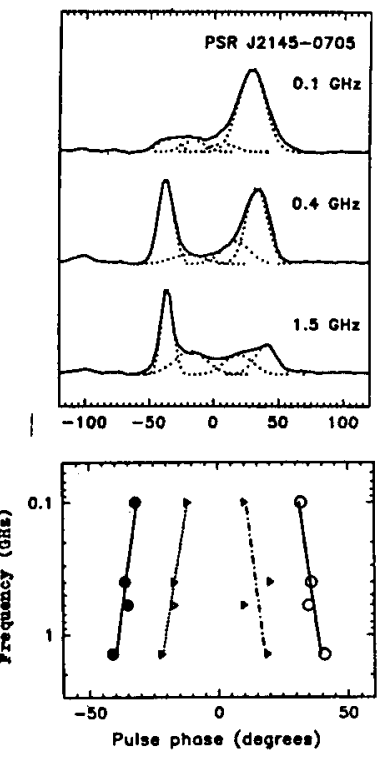

Figure 1. Gaussian fit to the integrated profiles at $0.1,0.4$ and 1.5 $\mathrm{GHz}$ assuming four component structure. Solid line- the observed profiles, dotted lines- the Gaussian components. Beneath is the frequency dependence of the phase position of components. Note that the scale in the lower graph is extended by 2 times. 
The result, illustrated in Fig.1., is that the separation between the components increases with frequency in contrast to the behavior seen usually in pulsars. For the most pronounced outermost leading I and trailing II components the separation increases with frequency as $S(f) \propto f^{+0.1}$. For the inner components III and IV the signal-to-noise ratio is lower and their parameters are less reliable. Nevertheless the frequency dependence of the separation has the same character: the separation between the components increases with frequency too.

We suggest, that these peculiarities may be interpreted as an effect of multipole components of the magnetic field. The dipole plus quadrupole magnetic field is illustrated in Fig.2. It is not symmetrical contrary to that of a dipole. In the lower part of Fig.2, where north polarity $n$ of the quadrupole is opposite to the South polarity $S$ of the dipole, the single dipole magnetic field cone at large radii will bifurcate at lower radii into two separate arches which diverge downwards and touch the star at two poles of the quadrupole of the same polarity, but of diametrically opposed location. As a result, at some height above the star the separation between components will increase as the radius decrease.

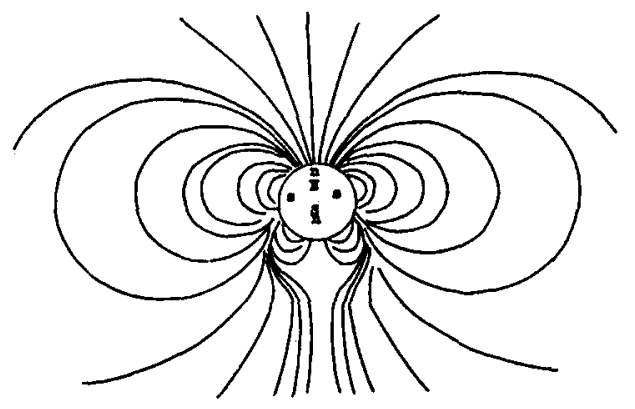

Figure 2. Computer simulation of the supperposed dipole and quadrupole magnetic field. $N$ and $S$ are the poles of dipole and $n$, $s, n$ and $s$ are the poles of quadrupole.

This scenario is easier to realize in short period millisecond pulsars, where the radius of the light cylinder is small and the emission region is closer to the surface, where the multipole field character is more pronounced.

\section{References}

Bailes M. et al. 1994, ApJ, 425, L41

Foster R.S., Fairhead L., Backer D.C., 1991, ApJ, 387, 687.

Wu Xinji, Xu Wenand, Rankin J.M., 1992, in Proc, IAU Symp. N 128, Pedagogical Univ. Press, p.172-175 